\title{
Bases de données prosopographiques, humanités numériques et histoire de la justice: les magistrats belges et coloniaux entre crises et modernisations judiciaires, 1795-1962
}

Xavier Rousseaux

Xavier Rousseaux : Xavier Rousseaux est directeur de recherches au Fonds National de la Recherche Scientifique (F.R.S.-FNRS), et professeur extraordinaire à l'Université catholique de Louvain, Louvain-la-Neuve, Centre d'histoire du droit et de la justice. Spécialiste de l'histoire de la justice et du crime, il dirige le Pôle d'attraction interuniversitaire Justice \& Populations. The Belgian experience in international Perspective, 1795-1950. Il a coédité les ouvrages suivants : Monuments ou documents ? Les comptabilités comme source pour l'histoire du contrôle social (XIIIe-XVIIIe siècle), éd. A. Wirth-Jaillard, A. Musin, N. Demaret, E. Bodart et X. Rousseaux, Bruxelles, AGR, 2015 (Studia 154) ; Tweehonderd Jaar Justitie. Historische Encyclopedie van de Belgische Justitie. Deux siècles de justice belge. Encyclopédie historique de la justice belge, éd. M. De Koster, D. Heirbaut et X. Rousseaux, Bruges, La Charte-Die Keure, 2015 ; Modernisation of the Criminal Justice Chain and the Judicial System. New Insights on Trust, Cooperation and Human Capital, A. Hondeghem, X. Rousseaux et F. Schoenaers, Springer, 2016; Policing New Risks in Modern Europe History, éd. J. Campion et X. Rousseaux, Basingstoke, Palgrave-Mac Millan, 2016; Mons dans la tourmente. Justice et société à l'épreuve des guerres (1914-1961), éd. A. Dumont, A. Thiry, J. Campion et X. Rousseaux, Louvain-la-Neuve, Presses Universitaires de Louvain, 2016.

DOI: $10.25518 / 1370-2262.510$

Mots-clés : Bases de données

relationnelles, Magistrature, Méthodologie, Prosopographie, Sociologie historique

Keywords : Historical sociology, Judiciary, Methodology, Prosopography, Relational databases

\section{Avant-propos}

À l'heure où beaucoup envisagent l'atterrissage en douceur de leur carrière académique, Jean-Pierre Nandrin accepta de piloter une entreprise originale et risquée : la création d'une base de données informatisée des magistrats belges depuis 1795. Originale, car les répertoires biographiques sur la Belgique contemporaine accessibles sous forme numérique n'étaient pas légion. Risquée, car le monde des historiens ne disposait alors que d'expertise et d'infrastructures limitées dans le domaine des bases de données. Durant huit ans, cette aventure allait l'amener, avec ses collègues de Namur et Louvain-la-Neuve, à plonger dans les arcanes de l'informatique et de la prosopographie, à travers un projet du Fonds de la recherche fondamentale et collective (FRFC) et un Pôle d'attraction interuniversitaire (PAI) fédéral. Informatique et prosopographie avaient réveillé en Jean-Pierre l'historien moderniste des structures sociales et économiques de sa jeunesse. Avec son étude du corps de la première magistrature belge de 1830-1832, il fut un des pionniers de l'étude contemporaine de la prosopographie, discipline auxiliaire familière aux historiens des périodes anciennes et médiévales. Quant aux innovations des bases de données relationnelles, le doctorant en histoire contemporaine en avait déjà goûté les séductions et tâté les écueils à travers le logiciel "Quatrième dimension», utilisé pour cette reconstitution des magistrats de l’Indépendance1. 
Bases de données prosopographiques, humanités numériques et histoire de la ju...

Le 31 octobre 2015, lors du premier colloque international du second PAI «Justice et Populations, l'expérience belge en perspective internationale, 1795-2015 », consacré à la « Modernisation de la chaine pénale et du système judiciaire », une session a été consacrée à la présentation des travaux suscités par cette entreprise. Jean-Pierre n'a pu hélas en voir l'aboutissement. Plusieurs de ses étudiants, collègues et amis et notamment deux de ses doctorantes contribuèrent alors à exploiter et relancer l'œuvre, à travers différents projets de recherche et l'amélioration de l'application informatique. Le dossier qui suit constitue une version française révisée des contributions à ce colloque, publiées en langue anglaise2. Il trouve tout naturellement sa place dans les cahiers du Centre de recherche en histoire du droit et des institutions dont Jean-Pierre était une des chevilles ouvrières.

\section{Réflexions sur un projet}

Sous ce vocable abscons de prosopographie se cache une approche de la réalité sociale par l'étude biographique 3 . Mais, à la différence de la biographie connue du grand public, centrée sur un individu généralement illustre 4 , la méthode prosopographique s'intéresse à un groupe socioprofessionnel qu'elle étudie à travers les trajectoires biographiques des individus et les multiples aspects de leur insertion dans la société (alliances, réseaux, engagements, positions, honneurs, patrimoine...).

Ce principe de biographie collective s'est largement inspiré de l'analyse des réseaux interpersonnels : formation, structuration, reproduction. Cette méthode s'appuie également sur les développements épistémologiques et technologiques qui rendent possible aujourd'hui l'automatisation à grande échelle de croisements de données, autrefois réalisés manuellement. Citons les théories des ensembles ou des graphes, l'étude des réseaux ou les progrès de l'informatique tant du point de vue matériel (capacité de calcul et extension du stockage) que logiciel, en particulier le développement des systèmes de gestion de base de données relationnelles (SGBD).

Ces évolutions récentes permettent un renouvellement de l'entreprise prosopographique dont les racines remontent à la fin du $19^{\mathrm{e}}$ siècle dans le cadre des grandes entreprises de publications de sources nationales. Renouvellement car certaines disciplines, en particulier l'histoire des périodes plus anciennes, pour lesquelles la documentation est rare, éclatée et fragile reposent depuis longtemps sur le fil conducteur des listes nominatives, enrichies de toutes traces possibles. Les spécialistes des périodes anciennes ou proto médiévales en sont familiers, disposant même de «prosopographies de collection » à prétention générale $\underline{5}$. Mais aujourd'hui la méthode ne consiste plus à produire des fichiers papier, enrichis par leur auteur au long d'une vie laborieuse, ni à la publication de volumineux dictionnaires, constamment annotés par des chercheurs anonymes au gré de leurs trouvailles individuelles. La recherche repose sur la création d'un instrument souple de collecte, d'organisation et d'enrichissement de l'information. Mises à disposition du public sur un site internet, de telles bases sont susceptibles d'accroissements par des milliers de chercheurs reliés par la toile. La prosopographie, devenue discipline à part entière dispose de ses manuels $\underline{6}$, de sa revuez $\underline{7}$ et de ses sites internet $\underline{8}$.

Pour l'Occident médiéval, la révolution de l'écrit à partir du $13^{\mathrm{e}}$ siècle $\underline{9}$ rend de telles entreprises générales impensables, mais la croissance documentaire permet la multiplication de «prosopographies spécialisées ». Une rapide analyse des définitions données dans les sites et ouvrages spécialisés met en évidence combien la méthode touche aujourd’hui des groupes sociaux 
contemporains (artistes, médecins, ingénieurs, scientifiques, enseignants, fonctionnaires...).

Parmi ces entreprises prosopographiques, les «gens de justice » ou «praticiens du droit » ont tout particulièrement intéressés les chercheurs, dans une optique de genèse longue de fonctions cruciales pour l'État moderne ( $\mathrm{du} 13^{\mathrm{e}}$ au $18^{\mathrm{e}}$ siècle). Magistrats 10 et avocats 11 ont ainsi donné lieu à plusieurs recherches d'envergure. Au carrefour de domaines historiques comme l'histoire du droit, l'histoire politique et l'histoire sociale et de sciences sociales comme la démographie, l'anthropologie sociale et la sociologie des élites, portée par les méthodologies évoquées ci-dessus, l'étude du monde des praticiens du droit présente plusieurs intérêts. Il s'agit d'une population intimement liée à l'usage de l'écrit depuis le Moyen-Âge et donc documentée par des traces nombreuses.

Ces praticiens font partie des élites religieuses et laïques, tant sous l'Ancien Régime que dans les sociétés bourgeoises qui s'imposent à partir du $19^{\mathrm{e}}$ siècle, et témoignent d'une permanence étonnante comme groupes socioprofessionnels et comme élites politiques tout au moins jusqu'au milieu du $20^{\mathrm{e}}$ siècle12. Ils regroupent tous ceux qui, peu ou prou, influent sur l'établissement des normes, la résolution des litiges et la régulation des conflits, pratiques essentielles du fonctionnement d'une société donnée. Abordé dans la longue durée occidentale, ce monde du droit joua encore un rôle capital dans la formation de l'État moderne. Les légistes du Prince et les parlementaires au Moyen-Âge $\underline{13}$, les hauts fonctionnaires des Pays-Bas habsbourgeois $\underline{14}$, les magistrats belges de la Révolution française à l'Empire 15 , les avocats dans la République française 16 ou la magistrature à la Libération17, sont autant de corps dont les activités ont puissamment contribué aux mutations de la société occidentale. À partir du $18^{\mathrm{e}}$ siècle, un mouvement de professionnalisation des métiers du droit se développe. Hervé Leuwers l'a montré pour les avocats18. Ce mouvement aboutit à cloisonner le monde du droit, en en réservant progressivement la pratique à des catégories précises. Ces «professions juridiques » (avocats et avoués; notaires, magistrats ou greffiers) se sont essentiellement définies par une formation de base commune : un diplôme universitaire en droit et des pratiques professionnelles partagées.

Une présence attestée dans la longue durée et une professionnalisation croissante rendent l'étude de la magistrature capitale pour comprendre la formation des élites en Occident. Elle est fondamentale pour comprendre les processus de formation d'autres populations plus diffuses (les avocats, les jurés) ou précisément définies par les pratiques du champ juridique (les « criminels », les victimes, les justiciables).

L'idée d'étudier la magistrature belge provient à la fois de l'évolution de la recherche sur le droit comme de l'actualité politique et sociale. Dans les années 1960 et 1970 a émergé une approche pluridisciplinaire sur le droit, dont un des apports est d'avoir attiré l'attention sur le rôle des pratiques (Law in action) dans la compréhension du droit (Law in book). Renouveler l'étude du droit, en en contextualisant les usages dans une société, revenait à déplacer l'accent de l'analyse des textes aux acteurs : en l'occurrence les juges. D’autre part, depuis les années 1980, la Belgique, a été secouée par une vague de scandales autour du fonctionnement de la justice, ayant entrainé un divorce croissant entre monde judiciaire, monde politique et «opinion publique ». Le thème de la défiance entre les populations et l'appareil judiciaire est devenu un objet de débat. Au cœur de ce débat, le caractère corporatiste de la magistrature a été mis en avant pour justifier son apparente fermeture aux évolutions de la société. Ces deux approches - par les pratiques et les acteurs - ont conduit les chercheurs - juristes, sociologues et bientôt historiens - à s'intéresser aux magistrats. 
Bases de données prosopographiques, humanités numériques et histoire de la ju...

Ce sont, en effet, posées les questions de l'origine de la formation de la magistrature belge et de la réalité de cette lecture corporatiste.

Après des travaux préparatoires, deux entreprises individuelles ont marqué un premier état des connaissances. Passionné d'histoire, Jacques Logie, vice-président du tribunal de commerce de Bruxelles a entrepris une thèse sur la magistrature des départements " belges » à l'époque de la «réunion à la France », lors des révolutions de la fin du $18^{\mathrm{e}}$ siècle. Défendue en 1995, il en publia la synthèse en 199819. D'autre part, Jean-Pierre Nandrin, licencié en histoire et candidat en droit, entreprit une étude novatrice sur la formation de la magistrature au début de la Belgique indépendante. Il en reconstitua les premières nominations (1832) et s'intéressa aux juges de paix jusqu'en 184820. Une première exploration avait été faite dans le cadre d'une recherche dans l'espace « belge » à travers les Révolutions (1780-1830)21. Il s'agissait d'identifier la place du personnel judiciaire dans les transformations des élites entre les révolutions de la fin du $18^{\mathrm{e}}$ siècle et la Révolution belge de 1830.

De la réunion de ces deux pionniers, hélas trop tôt décédés, avec de jeunes chercheurs du Centre d'histoire du droit et de la justice (CHDJ) à Louvain-la-Neuve et des Facultés Universitaires NotreDame de la Paix à Namur (UNamur), germa l'idée d'un projet audacieux : construire une base de données relationnelle sur la « prosopographie des magistrats belges au $19^{\mathrm{e}}$ siècle (1830-1914)». Ce projet initié par Jean-Pierre Nandrin (Université Saint-Louis, Bruxelles (USL-B)), Xavier Rousseaux (Université catholique de Louvain (UCL)) et Axel Tixhon (UNamur), dans le cadre du développement d'une histoire intégrée de la justice, a bénéficié du soutien du Fonds national belge de la recherche scientifique (FRS-FNRS) depuis 2005. Il a successivement permis d'engager plusieurs chercheures en 2005-2007 (Françoise Muller (UCL), Anne Roekens et Bénédicte Rochet ( UNamur)) ainsi qu'un informaticien (Xavier Cuvellier (USL-B)) pour la conceptualisation de l'information historique et la réalisation de l'outil. La première étape a été de dessiner l'architecture de la base, ce qui a été mené par Françoise Muller avec le soutien d'Aurore François et de Frédéric Vesentini (UCL-CHDJ). Grâce à un mandat postdoctoral du FNRS, Vincent Bernaudeau (Université d'Angers) a rejoint l'équipe de l'USL-B pour la faire bénéficier de son expertise prosopographique. Il a particulièrement pris en charge tous les volets - de la conception à la publication des actes - du colloque organisé à l'UNamur du 14 au 16 décembre 2006222. L'ambition du projet était de créer un outil spécifique, mais transposable à d'autres données prosopographiques et d'autres univers nationaux. Dans cette perspective, le colloque a voulu, à la fois, réunir des expériences prosopographiques sur des groupes différents, réfléchir sur les problématiques et les apports de ces recherches à la compréhension de la naissance et de la structuration d'identités socio-professionnelles dans la longue durée et réfléchir aux méthodologies sous-jacentes au développement de telles approches. C'est pourquoi la dernière journée du colloque a été élargie au Groupe de contact FNRS sur les «Sources et méthodes pour l'histoire du contrôle social, du Moyen-Âge à nos jours», rassemblant archivistes, praticiens et historiens. Sous la houlette de l'Archiviste général du Royaume de Belgique, Karel Velle, une première version de la base de données « Magistrats » y a été notamment présentée.

Le projet prosopographie s'est progressivement étendu en amont et en aval du $19^{\mathrm{e}}$ siècle. En amont par l'intégration des données recueillies par le regretté Jacques Logie dans le cadre de son doctorat à Paris-IV sur la magistrature belge sous la Révolution et l'Empire. Grâce au travail de Catherine Goffin (UNamur), le lien entre la magistrature au cœur de la Révolution belge de 1830 et sa paternité sous le Directoire, le Consulat et l'Empire (1795-1814) a pu être renoué23. En direction du $20^{\mathrm{e}}$ siècle, le projet initial s'est intégré dans un programme de recherche financé 
par la politique scientifique belge sur «Justice et société : histoire socio-politique de la justice en Belgique (1795-2005)». Il s'inscrivait dans le pôle dirigé par le professeur Jean-Pierre Nandrin à $l^{\prime}$ USL-B concernant l'histoire de la magistrature belge au $19^{\mathrm{e}}$ siècle et durant la Seconde Guerre mondiale.

Par ailleurs, un deuxième mandat postdoctoral du FNRS a permis d'engager David Niget (Université d'Angers, Université du Québec à Montréal) dans l'équipe de l'UCL pour développer un axe complémentaire sur une magistrature spécifique : les juges pour enfants, créés dans la première moitié du $20^{\mathrm{e}}$ siècle.

Avec le développement de projets similaires, notamment la base de données sur les magistrats français de Jean-Claude Farcy느, le projet a fait l'objet d'interventions à des colloques spécialisés dans cette discipline émergente que sont les « humanités numériques » 25 .

$\mathrm{Au}$ gré de recherches ponctuelles, la base de données explora encore de nouvelles dimensions. La magistrature militaire belge depuis 1830 a ainsi fait l'objet de travaux complémentaires, grâce aux recherches d'Éric Bastin 26 et de Laurence Montel. Après une étude exploratoire menée par Laurence Montel et Enika Ngongo, un nouveau projet ambitionne, depuis juillet 2014, d'y intégrer l'ensemble des «magistrats » de l'Afrique coloniale sous influence «belge », de l'État Indépendant du Congo à la fin du mandat des Nations-Unies sur le Ruanda-Urundi.

En octobre 2017, on recense dans la base de données : 7567 personnes (dont 4647 magistrats belges des cours et tribunaux en Belgique et en Afrique), 142 nominations et plus de 5600 références 27. Par ailleurs, 854 juridictions ont été répertoriées et leur personnel replacé sur la ligne du temps.

$\mathrm{Au}$ cours de ces années, l'essentiel de l'énergie a été consacré à la récolte de l'information au travers des différentes sources produites : répertoires officiels, presse spécialisée ou archives. Si la récolte et la validation des informations ont pris du temps, et que les aspects techniques ont dévoré pas mal d'énergie, l'exploitation du matériau réuni a désormais commencé.

Si l'on tente un rapide parcours des projets disponibles, force est de constater le petit nombre de projets spécifiques actuellement disponibles en ligne sur les magistrats judiciaires pour les $19^{\mathrm{e}}$ et $20^{\mathrm{e}}$ siècles.

En France, « l'Annuaire rétrospectif de la Magistrature» de Jean-Claude Farcy (2010), signalé plus haut, se veut une mise en ligne des données conservées dans les fiches de carrière du Ministère de la Justice depuis 1827 jusqu'en 1987 en France métropolitaine et dans les colonies. Aux Pays-Bas, «Rechters in 1811 » recense 400 magistrats au moment de l'intégration du Royaume de Hollande au grand Empire français, identifiés à partir de sources locales28. Ces trois entreprises sont liées à des configurations différentes. La première en date, "Rechters in 1811 », est un élément de la thèse de doctorat de Maarten van Boven sur la formation des institutions judiciaires néerlandaises sous Napoléon29. "L'Annuaire rétrospectif » est davantage une application qui vise à donner accès à une source principale : les fiches de carrière des magistrats français. Alors que ces deux entreprises sont menées dans un cadre individuel, la base de données «Belgian Magistrates » est, dès le départ, une entreprise collective croisant plusieurs sources publiées et inédites autour de populations spécifiques : les magistrats judiciaires du Royaume et de ses colonies. Ces trois bases permettront-elles de renouveler l'histoire sociale des institutions contemporaines ? Pour la Belgique, des entreprises de fond permettent de l'espérer $\underline{30}$. Leur ambition est en tout cas de 
Bases de données prosopographiques, humanités numériques et histoire de la ju...

servir de point de départ accessible pour de nouvelles questions de recherche.

Les chapitres qui suivent présentent quelques sondages approfondis sur des périodes ou des thématiques exploitables à travers les sources mobilisées ainsi que l'application. La période de formation de la magistrature belge est étudiée par Emmanuel Berger dans la première contribution de ce dossier. À l'heure où la justice belge rompt avec le découpage issu de l'annexion à la France révolutionnaire, l'étude des relations entre magistrats « belges » et français, de l'accusation et du siège, met en évidence les tensions au cœur de cette circonscription nouvelle qu'est l'arrondissement judiciaire au début du $19^{\mathrm{e}}$ siècle.

Le deuxième chapitre rédigé par Laurence Montel, Pascaline le Polain, Bérengère Piret et Enika Ngongo s'attache à analyser l'entreprise de colonisation du Roi Léopold II en Afrique, sous l'angle du développement d'une magistrature «belge ». Aux côtés d'autres magistrats européens, les magistrats belges fondent un système judiciaire colonial inspiré de la justice métropolitaine et développent une culture propre qui pourra en retour inspirer la magistrature métropolitaine.

Les tensions vécues par la magistrature lors d'une situation inédite d'occupation militaire et civile lors de la Première Guerre mondiale servent de laboratoire pour étudier les rapports entre le droit et le pouvoir, entre ordinaire et extraordinaire dans la troisième contribution, rédigée par Mélanie Bost et Kirsten Peters. Inédite dans l'Europe du $20^{\mathrm{e}}$ siècle, la première occupation pose les jalons d'un modus vivendi entre magistrats occupés et autorités occupantes. La seconde occupation, vécue également par de nombreuses magistratures européennes, confronte les magistrats à un régime dictatorial. Dans l'ensemble cependant, la cohésion corporative de la magistrature belge lui permet de mieux résister aux volontés d'ingérence de l'occupant.

Ces trois enquêtes sur des périodes internationales de la magistrature belge ont été rendues possibles par les recherches intégrées dans l'application. Les questions de recherche ont nourri les orientations de la base, tandis que l'outil a permis de dépasser le cas individuel pour évaluer les tendances de fond et les exceptionnalités. Les potentialités et les développements futurs de celle-ci constituent le dernier exposé de ce dossier. Les auteures, Aurore François et Françoise Muller, évoquent les orientations qui ont été données à cette entreprise. D'une part, l'intérêt des technologies de bases de données et d'accès internet est de faciliter l'accès à des données publiques existantes mais longues et difficiles à manipuler. L'identification des individus, leur âge, sexe, lieux et dates de naissance et de décès permet de reconstituer des groupes proches et des générations. La recomposition des carrières va de pair avec la reconstruction des institutions dans lesquelles ils ont œuvré. Il en ressort des trajectoires individuelles et des institutions évolutives. Enfin, les productions intellectuelles constituent un autre champ de données publiques, dont l'accès est facilité et contextualisé par son lien à la base.

La multiplication des systèmes d'information numériques a, d'autre part, renforcé la protection de la vie privée, voire créé un «droit à l'oubli » numérique. Ceci a amené les concepteurs de la base à distinguer les données nécessaires à une recherche libre et cohérente, des données mises à disposition du "grand public ». Comme l'exige la recherche et le sanctionne la Commission nationale de Protection de la vie privée, des données plus sensibles à la vie privée sont recueillies, structurées mais ne feront pas l'objet d'une publication numérique ou papier. Ces données ne feront donc l'objet d'exploitation que sous forme quantitative ou anonymisée.

L'analyse de ces différentes entreprises biographiques de magistrats, comme les études de cas 
spécifiques de ce volume, éclairent bien certaines questions méthodologiques soulevées par Claire Lemercier et Emmanuelle Picard dans un ouvrage consacré aux approches biographiques et prosopographiques en histoire des sciences 31 . Celles-ci pointent les différences avec le recueil de données nominatives pour des périodes où l'information est rare et dispersée et les périodes plus contemporaines. Au-delà de l'identification d'individus dans des bribes documentaires, les chercheures évoquent trois grands ordres de contraintes et de possibilités des bases prosopographiques pour les périodes plus richement documentées, et particulièrement les $19^{\mathrm{e}}-21^{\mathrm{e}}$ siècles :

- La première réflexion porte sur la nécessaire définition du groupe sous examen. «Mais en situation d'abondance et d'hétérogénéité des sources, le regroupement des informations ne peut se faire de façon féconde que s'il s'appuie sur des hypothèses de recherche fortes, qui orientent tant la définition du groupe à considérer que la nature des renseignements à obtenir : il s'agit d'abstraire au moins autant que de décrire »32. Dans ce cadre, poursuivent-elles, définir le groupe est une opération cruciale. Dans le cas de la magistrature de type napoléonien, le bornage de la catégorie n'est pas trop malaisé. Il est produit par le processus de rationalisation amorcé par la Révolution et consolidé dès l'an VIII. Pas étonnant qu'au-delà de leur diversité les bases néerlandaise, française et belge soient plus ou moins marquées par un caractère commun, reliées qu'elles sont, de près ou de loin, à cet événement fondateur. Le noyau dur est composé des magistrats des tribunaux d'instance et des cours supérieures, dont la formation juridique devient la marque de fabrique. Les membres du parquet sont par des critères équivalents assimilables à ce corps reconstitué33. Le cas des juges de paix constitue une limite instructive, puisque de notables locaux au début du $19^{\mathrm{e}}$ siècle, ils sont progressivement intégrés au corps judiciaire, par la voie du diplôme et de l'examen. Restent les liens poreux entre une sphère plus large - celle des avocats - et le monde judiciaire. Les premiers, également identifiés par leur diplôme et l'examen, repeuplent les juridictions après l'essai révolutionnaire d'une justice sans juristes. Ils peuvent siéger comme juges suppléants ou assesseurs. En tout état de cause, dans les sociétés contemporaines, la professionnalisation de la sphère du droit se marque par la possession du diplôme de licencié en droit, sésame indispensable à l'entrée dans la carrière, manifestée par l'examen des candidatures par le ministère. Il n'en va pas de même des magistratures coloniales, où les situations sont déterminées par la pénurie des moyens disponibles. Ainsi pour la magistrature dans les territoires colonisés faut-il distinguer les magistrats de "carrière », dont le corps se modèle sur la magistrature métropolitaine, des agents territoriaux, administrateurs exerçant des fonctions de ministère public ou de juge d'instance, en lien avec leurs tâches administratives. Tout comme les juges de paix, ceux-ci sont d'abord recrutés sans formation spécifique. Sous l'État indépendant du Congo (1885-1908), beaucoup sont des militaires 34 , mais progressivement, le recrutement d'agents civils se professionnalise au travers du passage par des Écoles coloniales de statut universitaire $\underline{35}$.

- Le deuxième ordre de réflexion proposé par les auteurs porte sur les enjeux de la démarche prosopographique. Si définir le groupe est une opération indispensable, les objectifs de la prosopographie doivent également être mûrement réfléchis. Le plus souvent, « il s'agit de mettre en contexte l'institution et/ou la prise de position, scientifique par exemple, dans le social, les dispositions sociales, la famille - voire, à l'extrême, de les y dissoudre, de révéler une infrastructure qui rendrait plus ou moins vaine toute étude de la superstructure». Le fil conducteur des hypothèses le plus fréquent est que les caractéristiques externes 
Bases de données prosopographiques, humanités numériques et histoire de la ju...

à l'activité des individus (capital économique ou symbolique, familial, position sociale) déterminent les trajectoires de l'individu dans l'institution et les modalités collectives d'action de celle-ci. Dans le cas de la magistrature «napoléonienne », l'intérêt d'une base est tout d'abord de permettre l'entrée par les individus, en liant biographie individuelle et trajectoire dans l'institution, et à rebours de pouvoir partir de l'institution à un moment donné: par exemple une cour d'appel, ou une justice de paix et d'en mesurer l'homogénéité ou la diversité de composition dans l'instant et dans la durée. Dans une telle approche, la «superstructure » ne se lit qu'à travers l'« infrastructure » et l'individu (le magistrat) est en rapport dialectique avec le collectif proche (son tribunal). En termes bourdieusiens, «l'objet d'étude est alors non pas tant les individus que l'histoire et la structure du champ : réunir des informations sur un ensemble de caractéristiques individuelles permet de mieux comprendre cette histoire et cette structure $» \underline{36}$. Du point de vue de l'étude des institutions, en effet, la prosopographie a souvent été perçue comme un moyen de dépasser les organigrammes et les textes normatifs, pour injecter du «social » et de l'action collective dans le «juridique », ce qui pour la magistrature judiciaire apparait fondamental. En l'occurrence il s'agit d'interroger comment s'est constitué un « champ » judiciaire particulièrement compact et d'étudier quelle place il a occupée dans la gouvernance et la société.

- La comparaison entre groupes est la troisième problématique abordée par les auteures. Mesurer la cohésion sociale d'un groupe, son degré d'ouverture, sa capacité de mobilité sociale, le taux de féminisation ou son éventuel vieillissement n'a de sens que par comparaison avec d'autres groupes ou des données sur la représentativité du groupe sur la population globale. Ainsi, pour prendre un critère simple, la prosopographie des magistratures invite à prendre la mesure du taux de diplômés en droit parmi la population universitaire, et plus globalement, du pourcentage des diplômés dont la carrière s'effectue dans la magistrature, par rapport à ceux qui font carrière au barreau ou dans la politique37. Dans le cas belge, leur place dans les cadres politiques du nouvel État, issu de la Révolution de 1830 n'est pas négligeable, surtout lorsque l'on prend en compte le caractère fondateur de la loi d'organisation judiciaire de 1832 dans la structure de cet État38. Et cette place évolue fortement jusqu'au $21^{\mathrm{e}}$ siècle.

En fin de compte, pour le monde judiciaire, l'intérêt d'une telle entreprise n'est pas uniquement scientifique. Comprendre la part des logiques collectives et celle des compétences individuelles ou mesurer le fonctionnement d'une institution en période de démarrage, de crise ou de croisière, permet également de mieux piloter les hommes et les structures qui font l'État, ce que les démocraties avancées semblent avoir beaucoup de mal à mener. Dans le cas belge, la commission parlementaire chargée de l'enquête sur l'enquête dans l'affaire Dutroux avait mis en évidence les pratiques du quotidien des policiers comme des magistrats. Au-delà d'une dénonciation populiste des incompétences individuelles ou de la promotion de modèles managériaux venus du monde de l'entreprise, l'apport d'une sociologie historique de la magistrature est de mettre également en évidence combien une décision actuelle peut s'inscrire dans les ornières du fonctionnement du passé (path dependance), autrement dit comment la mémoire du passé peut définir l'agir du présent, en éclairant la crise supposée de la justice dans la société du début de $21^{\mathrm{e}}$ siècle.

\section{Notes}

1 J.-P. NANDRIN, La justice de paix à l'aube de l'indépendance de la Belgique (1832-1848). La 
professionnalisation d'une fonction judiciaire, Bruxelles, Publications des Facultés universitaires Saint-Louis, 1998.

2 Modernisation of the criminal justice chain and the judicial system. New insights on trust, cooperation and human capital, éd. A. Hondeghem, F. SchoenaerTs et X. RousseauX, Springer, 2015 (Ius Gentium, Comparative Perspectives on Law and Justice, vol. 50). Plus précisément : X. Rousseaux, Prosopography, Crisis and Modernisation of Justice - «Belgian Magistrates », an Introduction, p. 175-180 ; A. FranÇOIS et F. MUlLER, Prosopography in the Digital Age : Current Situation, Prospects and Perspectives in the Light of the Forthcoming "Belgian Magistrates » Application, p. 181-194 ; E. BERGER, Conflicts, Tensions and Solidarity Within the Judicial District : A Social-Professional Study of the Judiciary of the « Belgian » Departments Under the French Directory (1795-1799), p. 195-210 ; L. Montel, E. Ngongo, B. Piret et P. Le Polain DE WARouX, Magistrates of Congo (1885-1960). Prosopography and Biography as Combined Tools for the Study of the Colonial Judicial Body, p. 211-232 ; M. Bost et K. PETERS, Belgian Magistrates and German Occupiers : A Diachronic Comparison (1914-1918/1940-1944), p. 233-260. Ce dossier a été réalisé dans le cadre du PAI 7/22 Justice \& Populations. The Belgian experience in international perspective, 1795-2015.

$\underline{3}$ L. STONE, Prosopography, dans Daedalus, vol.100, 1971,p. 46-79.

4 Mentionnons cependant la tentative originale et controversée d'Alain Corbin sur la biographie d'un anonyme Le Monde retrouvé de Louis-François Pinagot. Sur les traces d'un inconnu, 1798-1876, Paris, 1998, rééd. Flammarion, 2002 (Champs) et les réflexions méthodologiques de J.-L. MAYAUD, «Recherches pinagotiques ». À propos du Monde retrouvé de Louis-François Pinagot, dans Ruralia [En ligne], n³, 1998, mis en ligne le 1 er juin 1998, consulté le 22 août 2014. URL : http://ruralia.revues.org/60.

5 J. MAURIN, La prosopographie romaine : pertes et profits, dans Annales. Économies, Sociétés. Civilisations, 1982, 35-5, p. 828. Citons parmi quelques exemples de ces entreprises générales : La Prosopographia Imperii Romani (PIR), The Prosopography of Later Roman Empire (PLRE), The Prosopography of the Byzantine Empire/World, la Prosopographie chrétienne du bas Empire et the Prosopography of Anglo-Saxon England, couvrant la période de 260 à 1261 pour le bassin méditerranéen et jusqu'à 1066 pour l’Angleterre.

6 Prosopography Approaches And Applications: A Handbook, éd. S. KeATS-RoHAN, Oxford, 2007 (Prosopographica \& Genealogica 13).

I Prosopon: The Journal of Prosopography, 1994-2002, 2006, http://users.ox.ac.uk/ prosop/ prosopon/prosopon.htm, [accédé le $1^{\mathrm{er}}$ octobre 2017].

8 http://prosopography.modhist.ox.ac.uk/directory.htm [accédé le $1^{\mathrm{er}}$ octobre 2017].

9 P. BERTRAND, Les écritures ordinaires : sociologie d'un temps de révolution documentaire : entre royaume de France et Empire, 1250-1350, Paris, Presses Universitaires de la Sorbonne, 2016.

10 F. AUTRAND, Prosopographie et genèse de l'état moderne, actes de la table-ronde CNRS et ENSJF, Paris, 22-23 octobre 1984, Paris, ENSJF, 1986 ; L'État moderne et les élites, apports et limites de la méthode prosopographique. Colloque international, octobre 1991, Paris, 1996 ; M. PopofF, Prosopographie des gens du Parlement de Paris (1266-1753) d'après les ms Fr. 7553, 
Bases de données prosopographiques, humanités numériques et histoire de la ju...

7554, 7555, 7555 bis conservés au Cabinet des manuscrits de la Bibliothèque nationale de France, 2 vol., Paris, Le Léopard d'Or, 2003 ; P. BAKs e.a., De Heeren van den Raede. Biografieën en groepsportret van de raadsheren van het Hof van Friesland, Hilversum, 1999 ; S. JAHNS, Das Reichskammersgereicht und seine Richter. Verfassung und Sozialstruktur eines höchsten Gerichte im Alten Reich, Köln, 2003-2004 (2 vol.).

11 R. HijBrecht e.a., Album advocatorum. De advocaten van het Hof van Holland, 1560-1811, Den Haag, s.d.

12 V. BERNAUdeAU, La justice en question. Histoire de la magistrature angevine au XIX ${ }^{e}$ siècle, Rennes, Presses Universitaires de Rennes, 2007 ; C. CHARLes, Les Élites de la République : 1880-1900, Paris, Fayard, 1987.

13 F. AUtRAND, Naissance d'un grand corps de l'État : les gens du parlement de Paris (1345-1454), Paris, 1981 (Publications de la Sorbonne).

14 C. Thomas, Le visage humain de l'administration. Les grands commis du gouvernement central des Pays-Bas espagnols (1598-1700), Bruxelles, Académie royale de Belgique, 2014 ;C.BRUneEL et J.-P. Hoyois, Les grands commis du gouvernement des Pays-Bas autrichiens. Dictionnaire biographique du personnel des institutions centrales, Bruxelles, Archives générales du Royaume, 2001.

15 J. Logie, Les magistrats des cours et tribunaux en Belgique, 1794-1814. Essai d'histoire politique et sociale, Paris-Genève, Droz, 1998.

16 G. LE BÉGUec, La République des avocats, Armand Colin, 2003.

17 A. BANCAUD, Une exception ordinaire. La magistrature en France, 1930 - 1950, Paris, Gallimard, 2002.

18 H. LEUWERS (éd.), L'invention du barreau français, 1660-1830. La construction nationale d'un groupe professionnelParis, Éditions de l'École des hautes études en sciences sociales, 2006 ; id., Juges, avocats et notaires dans l'espace franco-belge. Expériences spécifiques ou partagées (XVIII ${ }^{e}$-XIX ${ }^{e}$ siècles), Bruxelles, Archives générales du royaume, 2010 (Justice \& société, 2).

19 J. LOGIE, op. cit.

20 J.-P. NANDRIN, La justice de paix à l'aube de l'indépendance de la Belgique (1832-1848). La professionnalisation d'une fonction judiciaire, Bruxelles, Publications des Facultés universitaires Saint-Louis, 1998. Id., Hommes et Normes. Le pouvoir judiciaire en Belgique aux premiers temps de l'indépendance (1832-1848), Louvain-la-Neuve, 4 vol. 1995 (UCL, Thèse de doctorat en histoire, inédite).

21 X. ROUSSEAUX et J.-P. NANDRIN, Le personnel judiciaire en Belgique à travers les révolutions (1780-1832). Quelques hypothèses de recherches et premiers résultats, dans Le personnel politique dans la transition de l'Ancien Régime au Nouveau Régime en Belgique (1780-1830), Kortrijk, Anciens Pays et Assemblées d’État, 1993, p. 13-69.

22 C. Goffin, Les magistrats dans les départements réunis sous le Directoire (an IV-an VIII) : corps social à part entière?, dans L'acculturation des modèles policiers et judiciaires français en 
Belgique et aux Pays-Bas (1795-1815), éd. E. BERGER, Bruxelles, Archives générales du Royaume, 2010, p. 99-107.

23 Les praticiens du droit du Moyen Age à l'époque contemporaine. Approches prosopographiques (Belgique, Canada, France, Italie, Prusse), éd. V. BERNAUdEAU, J.-P. NANDRIN, B. Rochet, X. Rousseaux et A. TIXHON, Rennes, Presses universitaires de Rennes, 2008.

24 Annuaire rétrospectif de la magistrature. $X I X^{e}-X X^{e}$ siècles ; http://tristan.ubourgogne.fr:8080/[accédé le $1^{\mathrm{er}}$ octobre 2017].

$2 \underline{5}$ Université de Dijon, Journée d'étude : Base de données et histoire de la justice, 10 juin 2010, http://tristan.ubourgogne.fr/CGC/manifestations/09_10/10_06_10.html

$\underline{26}$ E. BAStin, La justice militaire en Belgique de 1830 à 1850. L'auditeur militaire, «valet » ou « cheville ouvrière » des conseils de guerre ?, Louvain-la-Neuve, Presses universitaires de Louvain, 2012.

$\underline{27}$ http://www.digithemis.be/index.php/applications/magistrats/acces [accédé le $1^{\text {er }}$ octobre $2017]$.

$\underline{28}$ www.members.home.nl/m.v.boven [accédé le 14 septembre 2017]. La dernière mise à jour du site date du 20 octobre 2015.

29 M. VAN BOVEN, De rechterlijke instellingen ter discussie. De geschiedenis van de wetgeving op de rechterlijke organisatie in de periode 1795-1811, Nijmegen, 1990.

$\underline{30}$ Pour nous limiter aux magistrats, citons à titre d'exemple les travaux de Françoise MULLER, La Cour de Cassation belge à l'aune des rapports entre pouvoirs, Bruges, La Charte, 2011 ; la thèse de Mélanie Bost, Traverser l'occupation 1914-1918. Du modus vivendi à la grève, la magistrature belge face aux occupants allemands, Louvain-la-Neuve, 2013 (UCL, thèse de doctorat en histoire inédite) et le doctorat, en cours, d'Amandine Dumont sur les magistrats coloniaux belges.

31 C. LEMERCIER et E. PICARD, Quelle approche prosopographique ?, dans Les uns et les autres... Biographies et prosopographies en histoire des sciences, éd. P. NABONNAND et L. RoLLET, Nancy, Presses universitaires de Nancy, 2012, p. 605-630.

32 Ibid.

$\underline{33}$ J. BOURDON, La réforme judiciaire de l'an VIII, Rodez, Carrère, 1942.

34 L. Gann et P. Duignan, The Rulers of Belgian Africa, 1884-1914, Princeton, Princeton UP, 1979.

35 L. DE CLERCQ, L'administration coloniale belge sur le terrain au Congo (1908-1960) et au Ruanda-Urundi (1925-1962), dans Annuaire d'Histoire administrative européenne, 18, 2006, p. 187-210.

36 C. LEMERCIER et E. PiCARD, op. cit.

$\underline{37}$ À l'instar de leur place dans le système politique français : G. LE BÉGUEC, op. cit.

38 Nous renvoyons ici aux travaux de Jean-Pierre Nandrin sur le sujet et notamment J.-P. 
Bases de données prosopographiques, humanités numériques et histoire de la ju...

NANDRIN, Hommes et normes. Enjeux et débats du métier d'un historien, éd. P.-O. DE BROUX, A. Hendrick, F. Muller et B. Piret, Bruxelles, Presses de l’Université Saint-Louis, 2016, partie I.

PDF généré automatiquement le 2020-07-13 02:58:12

Url de l'article : https://popups.uliege.be:443/1370-2262/index.php?id=510 